\title{
Structure of the Planarian Central Nervous System (CNS) Revealed by Neuronal Cell Markers
}

\author{
Kiyokazu Agata ${ }^{1 *}$, Yukihiro Soejima ${ }^{1}$, Kentaro Kato', \\ Chiyoko Kobayashi', Yoshihiko Umesono ${ }^{1,2}$ and Kenji Watanabe ${ }^{1}$ \\ ${ }^{1}$ Laboratory of Regeneration Biology, Department of Life Science, Faculty of Science, \\ Himeji Institute of Technology, Hyogo 678-1297, Japan \\ ${ }^{2}$ Graduate School of Science Technology, Kobe University, Nada, Kobe 657, Japan
}

\begin{abstract}
Planarians are considered to be among the most primitive animals which developed the central nervous system (CNS). To understand the origin and evolution of the CNS, we have isolated a neural marker gene from a planarian, Dugesia japonica, and analyzed the structure of the planarian CNS by in situ hybridization. The planarian CNS is located on the ventral side of the body, and composed of a mass of cephalic ganglions in the head region and a pair of ventral nerve cords (VNC). Cephalic ganglions cluster independently from VNC, are more dorsal than VNC, and form an inverted U-shaped brain-like structure with nine branches on each outer side. Two eyes are located on the dorsal side of the $3^{\text {rd }}$ branch and visual axons form optic chiasma on the dorsal-inside region of the inverted U-shaped brain. The $6^{\text {th }}-9^{\text {th }}$ branches cluster more closely and form auricles on the surface which may function as the sensory organ of taste. We found that the gross structure of the planarian CNS along the anterior-posterior (A-P) axis is strikingly similar to the distribution pattern of the "primary" neurons of vertebrate embryos which differentiate at the neural plate stage to provide a fundamental nervous system, although the vertebrate CNS is located on the dorsal side. These data suggest that the basic plan for the CNS development along the A-P axis might have been acquired at an early stage of evolution before conversion of the location of the CNS from the ventral to the dorsal side.
\end{abstract}

\section{INTRODUCTION}

Planarians are considered to be among the most primitive animals which acquired the central nervous system (CNS), mesodermal tissues, and bilateral structure during evolution. It is also considered that planarians may branch from the common ancestors of both protostomes and deuterostomes since the mouth and the anus have not been segregated (SaliviniPlawen, 1978). Moreover, it has been suggested that planarian neurons more closely resemble the neurons of vertebrates than those of higher invertebrates (Sarnat and Netsky, 1985). So, it is interesting to analyze the structure and the genetic program of the planarian CNS for understanding the origin and evolution of the CNS. In particular, recent genetic studies suggested that common genes are involved in the brain development of both Drosophila and mouse (Simeone et al., 1992; Hirth et al., 1995; Thor, 1995). Although it is a fact that the otd gene of Drosophila can be replaced with the Otx-2 gene of human (Furukubo-Tokunaga, personal communication), it is still difficult to imagine the common genetic programs between them, since the position and the structure as

\footnotetext{
* Corresponding author: Tel. +81-7915-8-0187; FAX. +81-7915-8-0187.
}

well as the developmental processes are quite different between them. In this paper, we show the structure of the planarian CNS using several molecular markers, and we conclude that there is striking similarity in the structure of the planarian CNS along the anterior-posterior (A-P) axis with that of the "primary" CNS in early vertebrate embryos, which appears during early neurogenesis of vertebrates (Chitnis et al., 1995). These data suggest that the basic plan for CNS development along the A-P axis might have been acquired at an early stage of evolution.

\section{MATERIALS AND METHODS}

\section{Screening of neural marker genes}

A cDNA library containing $4 \times 10^{6}$ independent clones derived from the head region of a planarian, Dugesia japonica, was constructed using ZAPII as a vector. Two hundred cDNA clones were randomly picked and sequenced from the $5^{\prime}$ end. The nucleotide sequences and deduced amino-acid sequences were searched by the DDBJ online service using the fasta and tfsata programs, respectively. In situ hybridization screening was also done to find neural marker genes.

\section{In situ hybridization}

A clonal strain of Dugesia japonica established in our laboratory was used for in situ RNA hybridization. It was treated with $2 \% \mathrm{HCl}$ solution to remove the mucus for $5 \mathrm{~min}$, and then fixed in Carnoy's 
solution for whole-mount in situ hybridization or in formalin/picric acid/ glutaraldehyde mixture for paraffin section for $3 \mathrm{hr}$. For bleaching the sample, fixed samples were incubated in methanol containing $10 \%$ $\mathrm{H}_{2} \mathrm{O}_{2}$ under light for $10 \mathrm{hr}$ at room temperature. Hybridization was performed at $55^{\circ} \mathrm{C}$ in a solution containing $50 \%$ formamide, $5 \times \mathrm{SSC}$, $0.1 \%$ Triton $\mathrm{X}-100,0.1 \mathrm{mg} / \mathrm{ml}$ heparin, $1 \mathrm{mg} / \mathrm{ml}$ yeast tRNA and $10 \%$ Dextran sulfate with a DIG-labelled antisense RNA probe overnight. A mixture of BCIP/NBT was used for color development of the alkali phosphatase-conjugated anti-DIG-antibody (Boehringer).

\section{Observation of axon bundles}

We found that axon bundles could be easily distinguished when the sections which had been subjected to in situ RNA hybridization were viewed by dark field microscopy. However, this method is delicate, and not very reproducible. The degradation of the tissue during fixation may be critical to get clear results. We have sometimes used the $\mathrm{PH} 117$ probe to stain axon bundles in sections. This method is reproducible, but the specificity is not so high. This probe could not be used to observe axon bundles in whole-mount in situ hybridization.

\section{Immunohistochemistry}

Ascites fluid containing a monoclonal antibody against visual cells (K. Watanabe et al., submitted for publication) was diluted 1/20,000 and reacted with the sample after color development of in situ hybridization. A signal was detected using a peroxidase-conjugated secondary antibody (BioRad) in a solution containing 4-chloro-1-naphtol.

\section{RESULTS}

\section{Isolation of a neural marker gene}

To isolate a molecular probe staining planarian CNS, we sequenced 200 cDNA clones randomly picked from a head cDNA library of a planarian (Dugesia japonica). One of these clones, designated $\mathrm{PH} 04$, encoded a protein homologous to PC2 (Pro-hormone Convertase 2). This clone contained a 1.789 bp insert, and encoded a 525 amino-acid open reading frame which shows high similarity to PC2 of other animals (Fig. 1A). Amino acid sequences encoded by the PHO4 insert show more than $63 \%$ identity with PC2 of both invertebrates and vertebrates (Fig. 1B). However, it shows less than $43 \%$ identity with other PC families. Sequence analysis suggests that the $\mathrm{PHO} 4$ clone contains a partial cDNA fragment transcribed from the planarian PC2 gene. PC2 is known as a protease with processes precursor proteins of neuropeptides to mature forms, and is specifically produced in neurons secreting neuropeptides in other animals (Seidah et al., 1992; Ouimet et al., 1993).

Figure $2 \mathrm{~A}$ and $2 \mathrm{~B}$ show a horizontal section of the head region with histological staining and a horizontal section with in situ hybridization using the antisense PH04 RNA, respectively. Numerous cells which are considered to be neurons and/or other types of neural cells are clustered surrounding the inverted U-shaped axon bundles of the head region (Fig. 2A) and these cells are specifically stained by the PH04 RNA probe (Fig. 2B). There is no positive signal in other regions. We concluded that the $\mathrm{PH} 04$ probe can be used as a marker detecting neural cells in Dugesia japonica, although we could not identify what type(s) of neural cells were stained.

Regarding other clones, the $\mathrm{PH} 117$ probe stained the axon bundles strongly by in situ hybridization, although the cell-type specificity is low. This clone contains a about $1.5 \mathrm{~kb}$ insert. Deduced amino acid sequence encoded by the $\mathrm{PH} 117$

A

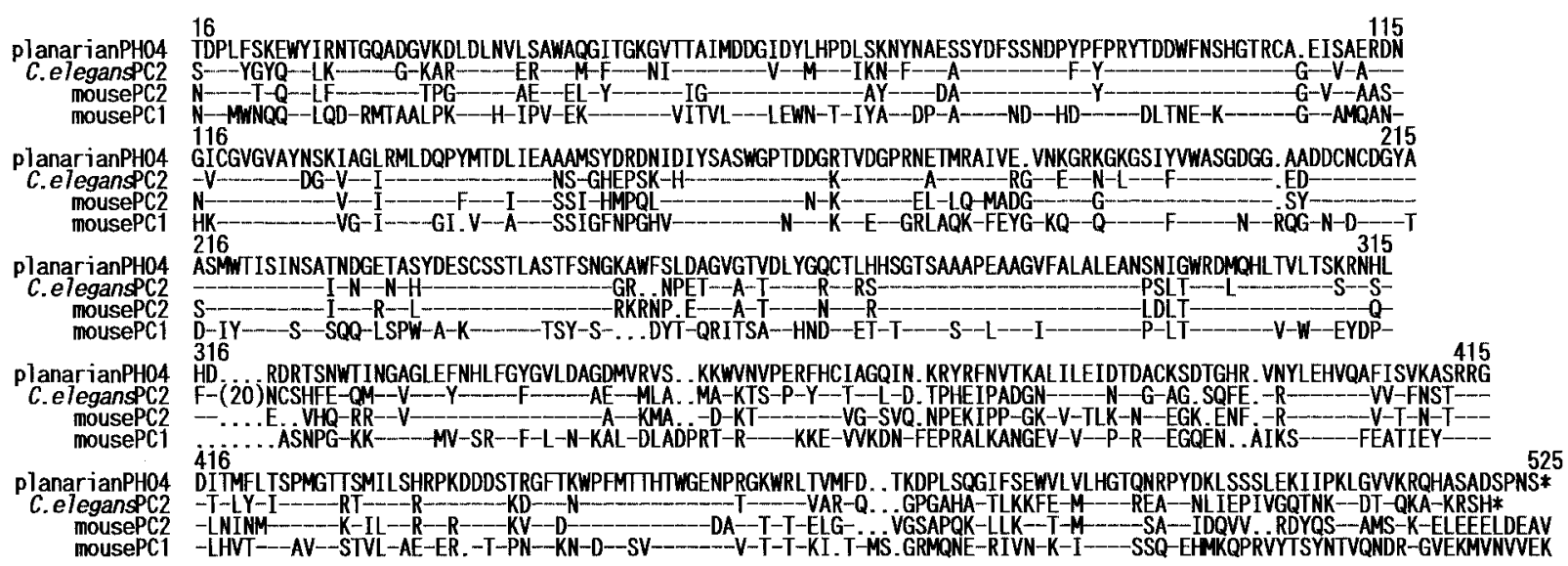

$\mathbf{B}$

\begin{tabular}{|c|c|c|c|c|c|c|c|c|c|}
\hline & C.elegansPC2 & aplysiaPC2 & amphioxusPC2 & XenopusPC2 & snai IPC2 & mousePC2 & mousePC1 & mousePC4 & mousePC5 \\
\hline planarianPH04 & $63.3 \%$ & $66.5 \%$ & $62.4 \%$ & $63.9 \%$ & $64.9 \%$ & $65.5 \%$ & $44.1 \%$ & $41.2 \%$ & $42.4 \%$ \\
\hline
\end{tabular}

Fig. 1. The $\mathrm{PHO} 4$ protein sequence and its similarity to $\mathrm{PC} 2$ of other animals. (A) Predicted protein sequence of the $\mathrm{PHO} 4$ insert, aligned with PC2 of $C$. elegans and mouse, and PC1 of mouse. Conserved amino acids are indicated by hyphens. Dots indicate deleted residues. (20) in the alignment of $C$. elegans indicates a 20 amino-acid insertion. Asterisks indicate termination. PC2 and PC1 of mouse have longer C-terminal regions than PC2 of the planarian and $\mathrm{C}$. elegans (data not shown). (B) Percentage of the identical amino acid residues between the PH04 protein and PC2, PC1, PC4 and PC5 of other animals. 

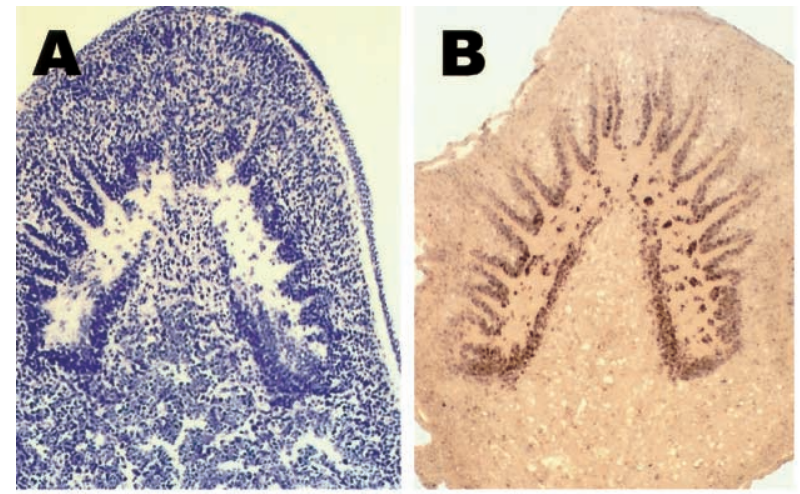

Fig. 2. Distribution of the $\mathrm{PH} 04$ positive cells. (A) Histological staining of a horizontal section of the head region. (B) In situ hybridization of a horizontal section of the head region by the $\mathrm{PH} 04$ antisense RNA probe.

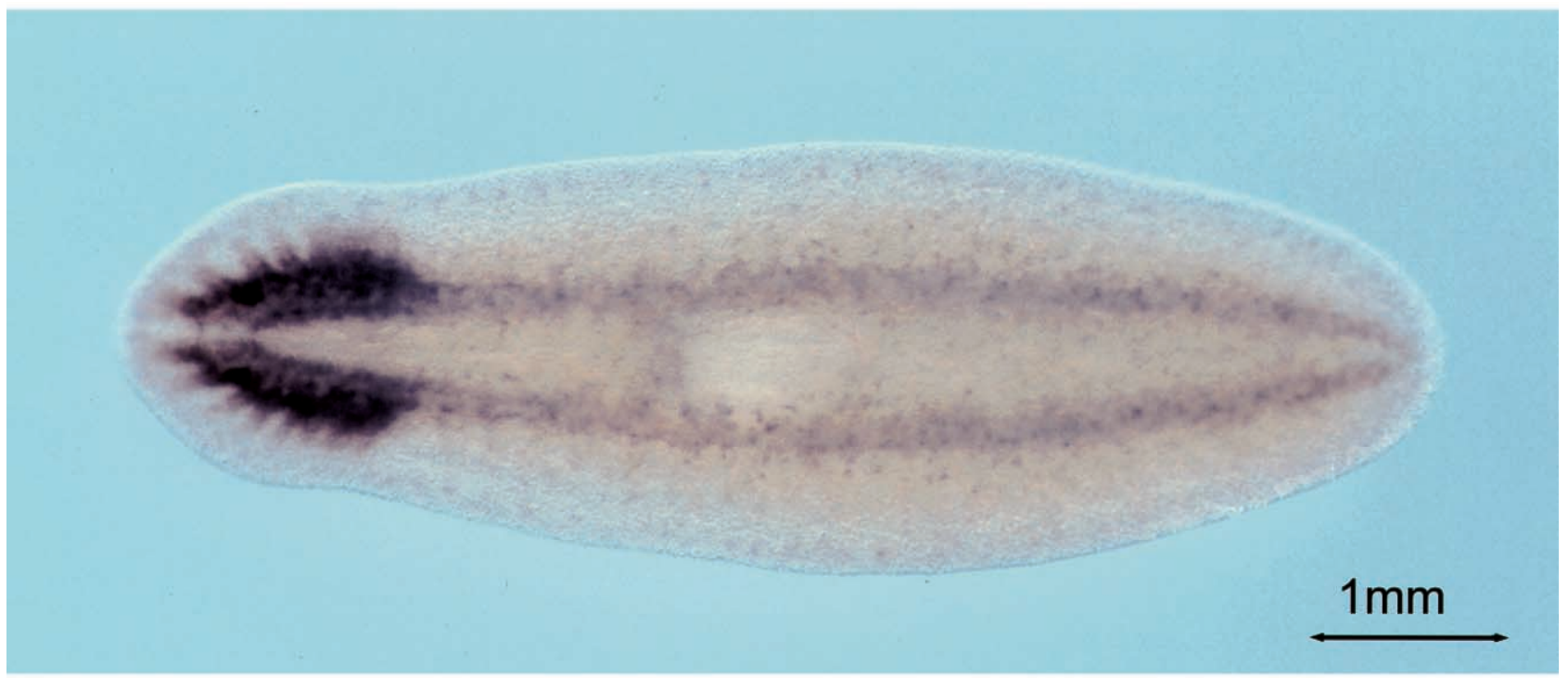

Fig. 3. Structure of planarian CNS. Ventral view of the whole mount in situ hybridization with the PHO4 antisense RNA probe. Anterior is left and posterior is right. The white region in the center of the body is a pharynx.
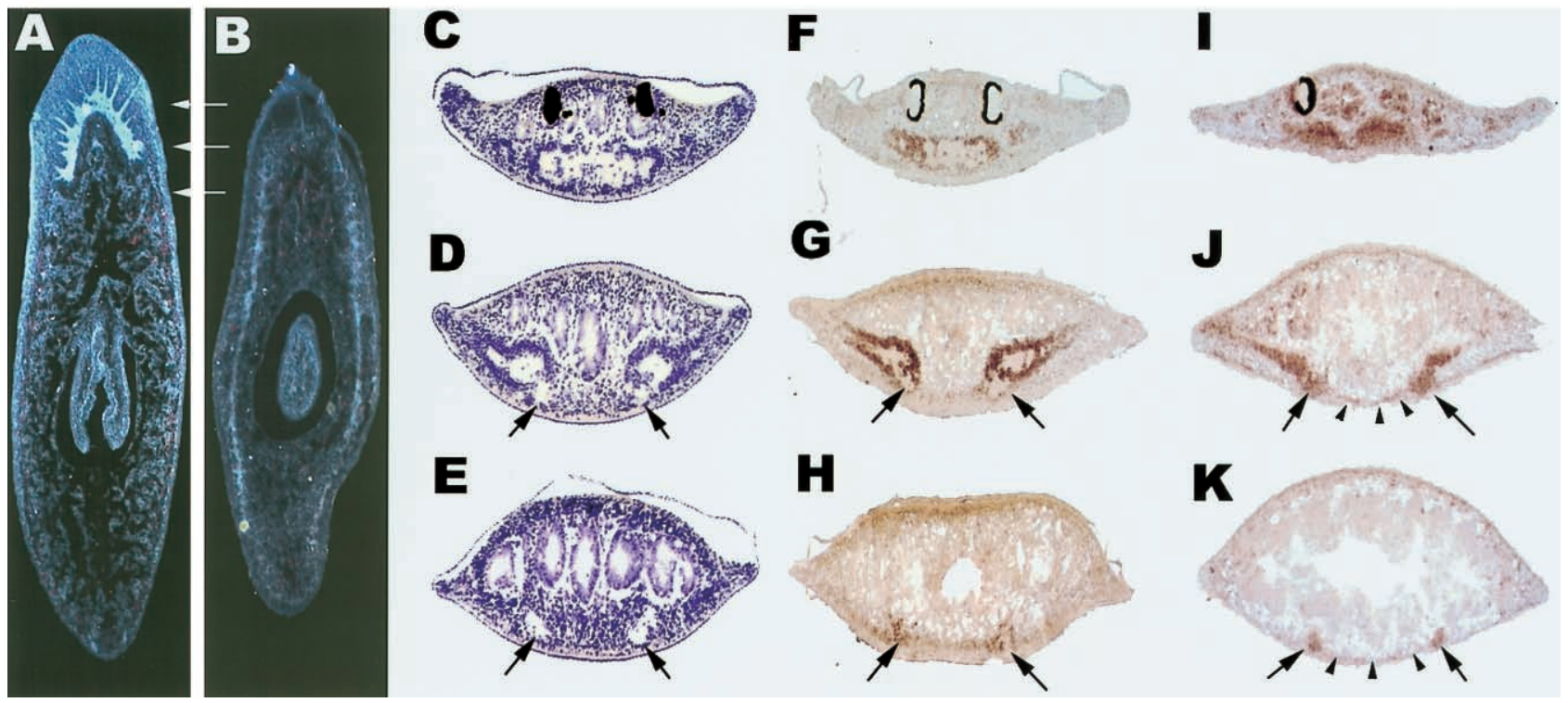

Fig. 4. Structural relationship between the brain and VNC. (A-B) Horizontal sections containing axon bundles surrounded by cephalic ganglions (A) and VNC (B). The pharynx is located in the center. (C-K) Transverse sections at the level of the head region (white arrows in A). (C-E) Cresyl Violet staining. (F-H) Distribution of PH04-positive cells. (I-K) Distribution of axon bundles stained by the PH117 probe. VNC and it's commisure axon are indicated by black arrows and arrowheads, respectively. 

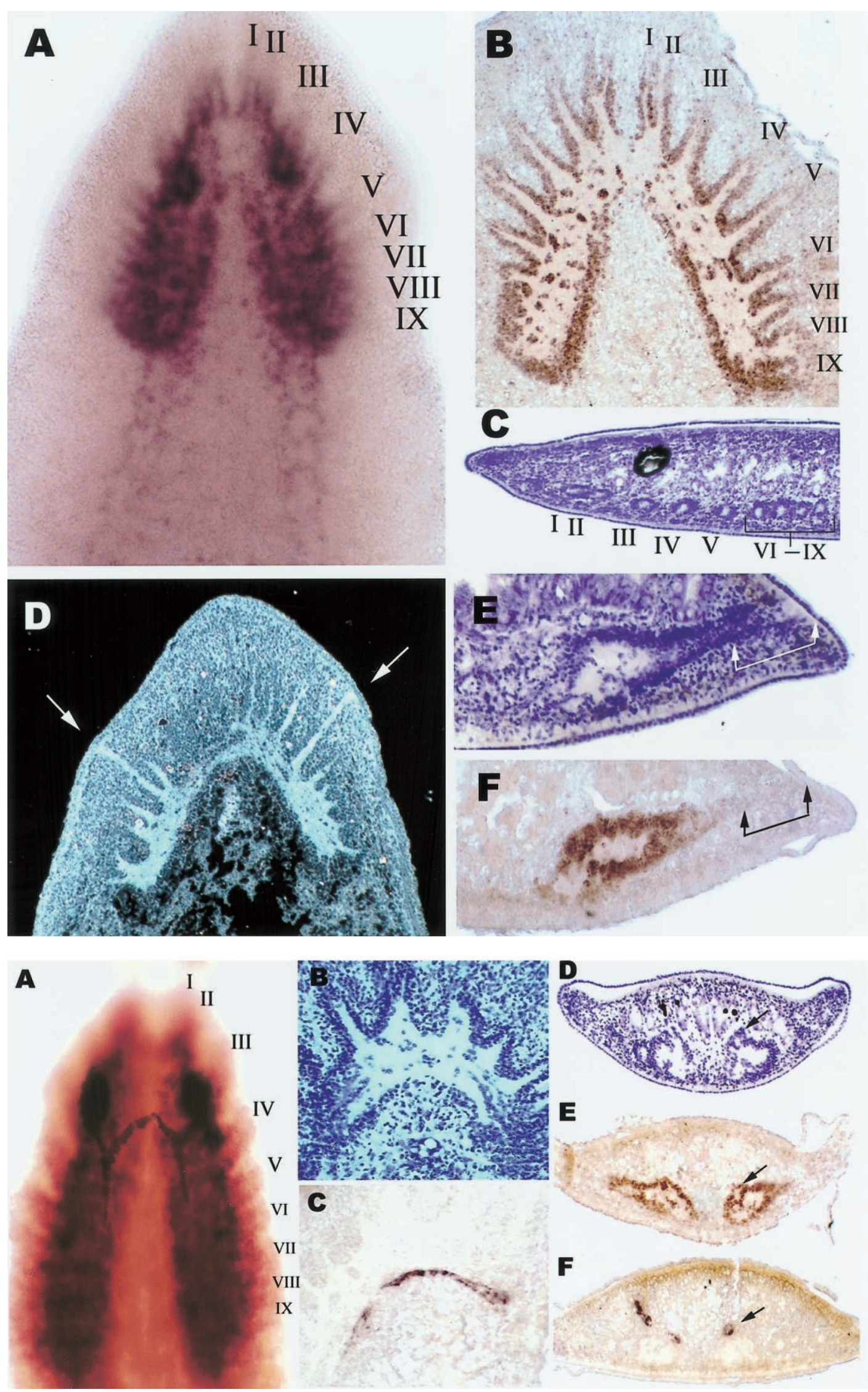
insert shows $72 \%$ identity with the human hsp40 (data not shown). We have sometimes used this probe to observe the axon bundles (see Fig. 4I-K).

\section{Structure of the planarian CNS}

To understand the structure and regeneration process of the planarian CNS, we have developed the whole-mount in situ hybridization method. We have succeeded in establishing a low background method by using Carnoy's fixation (see Materials and Methods). Figure 3 shows the ventral view of a whole-mount in situ hybridization using the antisense $\mathrm{PHO} 4$ RNA as a probe. It clearly shows that the planarian CNS is composed of a mass of cephalic neural cells in the head region and a pair of ventral nerve cords (VNC). Cephalic neural cells cluster in the anterior portion and form the inverted Ushaped structure.

To understand the structural relationship between the cluster of cephalic neural cells and VNC, we have analyzed their junction region by horizontal and transverse sections (Fig. $4 \mathrm{~A}-\mathrm{K})$. In the dark field views of horizontal sections, the inverted $U$-shaped axon bundle of the cephalic ganglion region is observed on a more dorsal section (Fig. 4A) rather than at the level of VNC (Fig. 4B). We could not observe any horizontal section that contained both cephalic neurons and VNC. The transverse sections indicated more clearly the relationship between cephalic neural cells and VNC. Figures, $4 \mathrm{C}-\mathrm{K}$ show the transverse sections of the anterior head region at the eye level (C, F and I), posterior head region (D, G and J) and trunk region ( $\mathrm{E}, \mathrm{H}$ and $\mathrm{K}$; see white arrows in Fig. $4 \mathrm{~A}$ ). The cephalic neural cells are located more dorsally than are the VNC. Many PH04-positive cells cluster closely together and form an independent structure on the dorsal side of VNC (Fig. 4G). There are few PH04-positive cells surrounding the VNC (Fig. 4H). The horizontal and transverse sections revealed that the cluster of cephalic neural cells forms a structure independent of VNC, and that VNC are connected to the cluster of cephalic ganglion cells on the most ventral-inside region of the inverted $U$-shaped structure in the head region. (Fig. 4D, G, J).

The interconnection system between left and right sides is also different for cephalic ganglions and VNC. The left and right VNC are connected by transverse axons between them. These transverse axons form a ladder structure from head to tail (Fig. 4K, arrowheads). However, no transverse axon is observed inside the inverted U-shaped structure. The left and right cephalic ganglions may be connected in the inverted $U$ shaped axon bundles (Fig. 4A). Although transverse axons are described in the head region in textbooks (Brøndsted, 1969), they are commissure axons connecting a pair of VNC, not connecting the cephalic neurons (Fig. 4J, arrowheads). These results indicate that the planarian CNS is composed of two independent structure: the cluster of the cephalic neural cells in the head region and a pair of VNC from head to tail, and they are connected to each other along the most ventralinside region of the cluster of cephalic neural cells in the head region.

\section{Structure of the planarian brain}

The segmental structure of the cluster of cephalic ganglions is observed only on the outside of the cluster (Fig. 5A,B,D). No segmental structure is observed on the inside of the inverted U-shaped structure. Nine branches on each side process to the surface of the head region (Fig. 5A-C). The terminals of the processes reach the head surface and form sensory organs (Fig. 5D,E, arrows). Of special note, the 6th-9th branches cluster more closely and form the auricles on the surface which function as chemoreceptors (Fig. 5A-C; Pigon, 1974). The neurons surrounding the tip of the process, which may be sensory neurons, are not stained by the $\mathrm{PH} 04$ probe (Fig. 5E, F, arrows).

In Fig. 6A, cephalic neuronal cells are stained brown by in situ hybridization with the antisense $\mathrm{PH} 04$ RNA, and the visual axons are stained purple by immunological reaction with an anti-visual-cell monoclonal antibody, VC-1. Some visual axons formed the optic chiasma in a planarian (K. Watanabe et al., submitted for publication). The eyes are located on the dorsal side of the $3^{\text {rd }}$ branch (Fig. 6A). The dorsal-inside region of the inverted U-shaped cluster of the cephalic ganglions forms the visual center and optic chiasma (Fig. 6A-C). The visual axons enter the visual center at the $3^{\text {rd }}$ branch level and then some of them project to the opposite side along the most dorsal-inside region. They terminate between the $4^{\text {th }}$ and $5^{\text {th }}$ branches (Fig. 6A). The bundles of the visual axons in the visual center are surrounded by $\mathrm{PH} 04$-positive cells (arrows in Fig. 6D-F). They form a neuromere-like compartment.

\section{Regeneration process of the brain}

To understand the regeneration process of the brain, we have removed both the head and tail portions and followed the regeneration process by in situ hybridization. Although we could not follow the brain-forming cells during the early stage of regeneration by in situ hybridization of sections, we could follow the early stage of brain regeneration by whole mount staining. Figure 7 shows the 2-day (left) and 4-day (right) re-

Fig. 5. Structure of the planarian brain. (A-B) Higher magnification views of the head region stained with the PH04 antisense RNA. Dorsal view of a whole mount staining $(\mathbf{A})$, and a horizontal section (B). Nine processes are numbered. (C) A parasagital section of the head region stained by Cresyl Violet. (D) Dark field view of a horizontal section. The processes which reach the surface are indicated by white arrows. (E-F) Higher magnification views of the transverse section. Cresyl Violet staining (E) and in situ hybridization (F). PH04-negative region is indicated by arrows. Fig. 6. Positional relationship between the visual axons and the brain. (A) Dorsal view of a whole mount double staining using the PH04 antisense RNA (brown) and anti VC-1 monoclonal antibody (purple). (B-C) Horizontal sections containing the visual center. Cresyl Violet staining (B) and immunohistochemistry of VC-1 (C). (D-F) Transverse sections containing the visual center. Cresyl Violet staining (D), in situ hybridization with the PH04 antisense RNA (E) and immunohistochemistry of VC-1 (F). The bundle of visual axons is indicated by an arrow. 


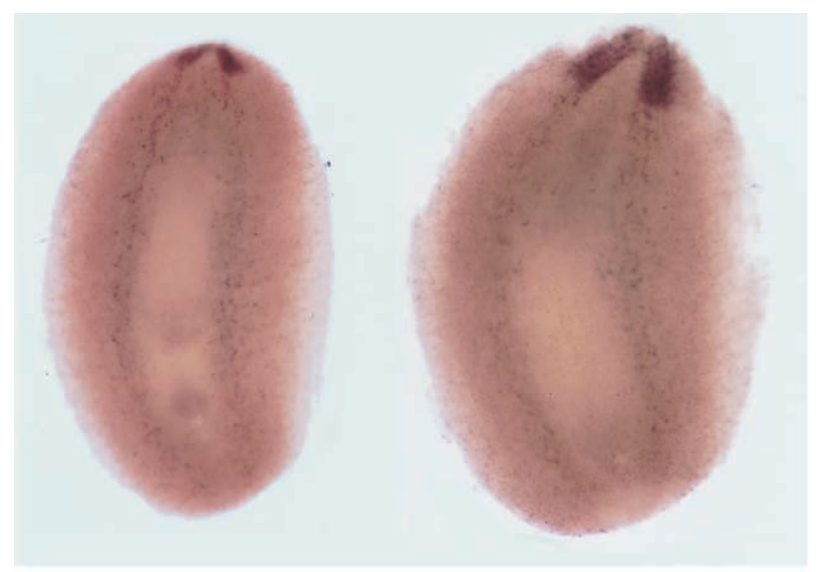

Fig. 7. Regeneration process of the brain. Ventral view of the whole mount in situ hybridization with the $\mathrm{PH} 04$ probe of the 2-day regenerate and the 4-day regenerate are shown in left and right, respectively.

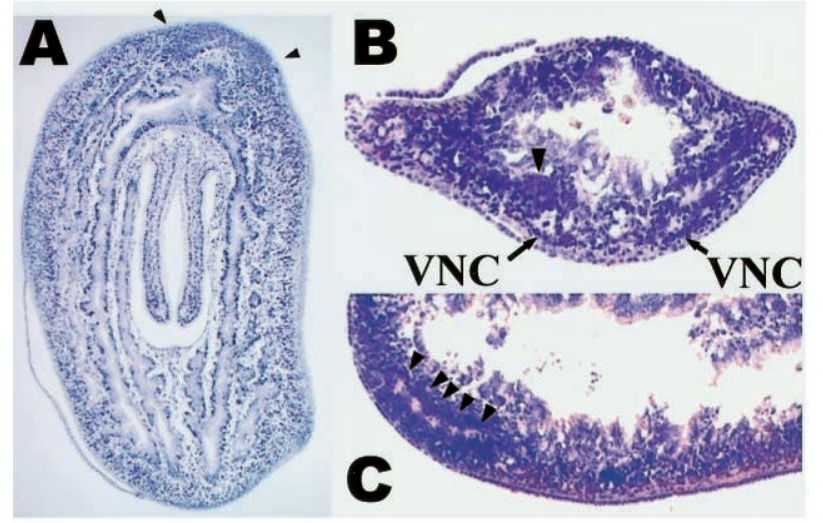

Fig. 8. Histological analyses of the regeneration process of the brain from the trunk fragment after removing the head and tail regions. (A) Horizontal section of the 2-day regenerate (Cresyl Violet staining). Blastema region of the anterior part is indicated by arrowheads. (B) Transverse section of the anterior part of the 2-day regenerate. The cell mass of the presumptive brain on the dorsal side of the remaining VNC is indicated by arrowhead. (C) Parasagittal section of anterior portion of the 4-day regenerate. Anterior is on the left. Five segments are indicated by arrowheads.

generates of the trunk fragments. Regeneration of the brain can be recognized in the most anterior portion of the regenerates within $48 \mathrm{hr}$ after amputation. Then, it grows gradually until it reaches the original proportion.

To investigate the origin of the cells forming the brain, the early regenerates were sectioned and stained by the $\mathrm{PH} 04$ probe. However, the signal was too weak to follow the PH04positive cells at early stages of regeneration by sections. Prospective cephalic neurons have already clustered in the dorsal portion of the remaining VNC of the 3-day regenerate (Fig. $8 \mathrm{~A}, \mathrm{~B}$, arrowheads), but we could not identify the origin of these cells . Our observations of the serial sections suggest that it is unlikely that the brain-forming cells derive from the neurons in the remaining VNC, since the presumptive brain is clustered in the space between the gut and VNC, and since no change of VNC was observed during regeneration (Fig.

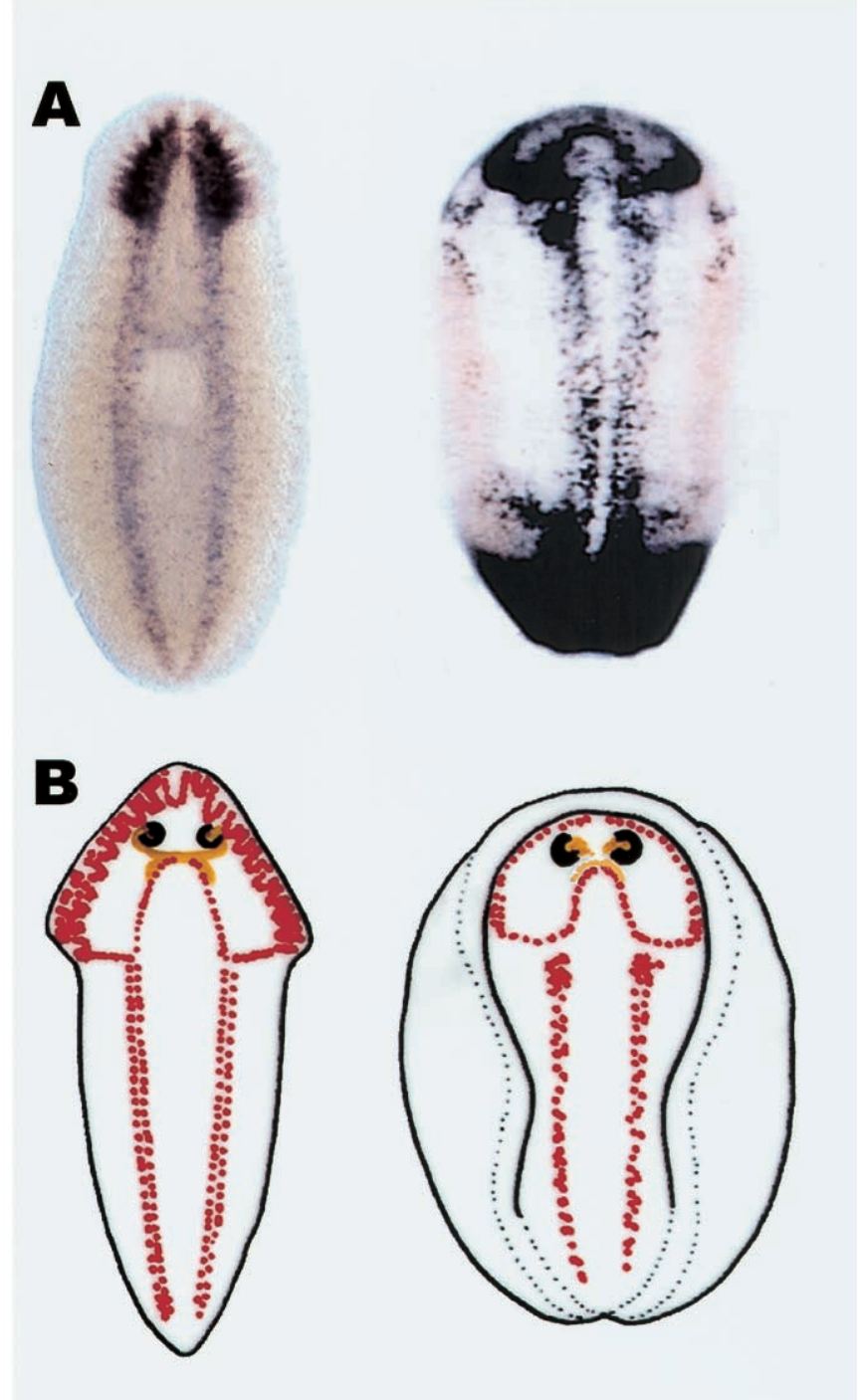

Fig. 9. Comparison of planarian CNS with the primary CNS of a Xenopus laevis embryo. (A) Left panel shows a ventral view of planarian CNS stained by the $\mathrm{PHO} 4$ gene as a probe. The whole mount view in Fig. 3 was reformed to become of a similar proportion to the Xenopus embryo. The right panel shows a dorsal view of the primary CNS of a Xenopus laevis embryo at stage 17 stained by $X$-Delta- 1 as a probe (reproduced from Chitnis et al., 1995, with permission from C. Kintner). (B) Schematic drawings of planarian CNS and the primary CNS of a Xenopus embryo at stage 14. PH04-positive neurons in the planarian and primary neurons of the Xenopus embryo are indicated in red. Two eyes and projection routs of visual axons of a planarian, and presumptive eyes and chiasma region of the Xenopus embryo are added onto the drawings.

$8 B)$. In the 4-day regenerate, segmentation of the lateral region of the brain was already observed, but only 5 branches were distinguished (Fig. 8C), suggesting that segmentation occurs unevenly in the process of regeneration. 


\section{DISCUSSION}

\section{Simple but well organized planarian CNS}

Here we have shown the structure of the planarian CNS by in situ hybridization using a PC2-homolog gene as a probe. Even though they are among the most primitive animals which develop the CNS, their CNS is better organized rather than we expected. Especially, in the head region, cephalic neural cells are well organized and form a brain-like structure. Visual axons project to the inner-dorsal region of the inverted $U$ shaped brain, and the visual center forms a neuromere-like compartment. Unfortunately, we have no probe identifying the sensory organs other than the eyes. If we obtain such marker molecules, it is expected that other subdomains in the brain will be distinguished.

It is currently of great interest to understand the cell-type complexity of neural cells in planarians. For example, the retina of planarians is composed of a single type of cell which processes not only microvilli into the pigment cup, but also an axon toward the visual center. That is, the single visual cell of planarians functions as a retinal ganglion cell as well as a photoreceptor cell. During evolution the cells have diversified into photoreceptor cells, interneurons, glial cells, and retinal ganglions, and formed a complex retina. This suggests that the cells comprising planarian brain may have the basic function(s) to process various external signals and retrace memories, but the composition of cells in the CNS may be simple. This may be one of the reasons why the structure of the planarian brain is simpler than that of higher animals.

\section{Regeneration process of the CNS}

Whole mount in situ hybridization analysis clearly indicated the early appearance of the regenerating brain. We could recognize a miniature of the brain within 2 days after amputation in the anterior tip of a trunk fragment. To understand why planarian can regenerate the brain but other organisms can not, it is important to identify the origin of the cells participating in brain regeneration. Observation of the serial sections suggested that prospective cephalic neurons appear from mesenchymal space, but not from the remaining VNC. However, we could not succeed in identifying the origin of the brainforming cells in this series of experiments.

\section{Striking similarity of the planarian CNS with the primary CNS of early vertebrate embryos}

We found that the structure of the planarian CNS is very similar to that of the primary CNS which is detected by the $X$ Delta-1 gene as a probe on the neural plate of Xenopus embryos (right panel in Fig. 9A; Chitnis et al., 1995), although the planarian CNS is located on the ventral side. A subpopulation of cells in the neural plate has already been differentiated as primary neurons expressing the $X$-Delta-1 gene. The distribution pattern of primary neurons shows striking similarity to the structure of the planarian CNS (Fig. 9A, B). Moreover, the relative positions of the eyes and optic chiasma in planarian brain are very similar to those of the presumptive eye and chiasma regions in the developing brain at this stage of Xenopus embryos (Fig. 9B; Li et al., 1997). These data suggest that the basic plan for CNS development along the A-P axis may have been established at an early stage of evolution. Although it is still not certain that we can compare the complex vertebrates brains to the invertebrate brains directly, it might be possible to compare the primary neurogenesis of vertebrates to the primitive CNS.

\section{New views about CNS development and evolution}

If pattern formation of primary neurogenesis is controlled under a genetic program for CNS development similar to that established at an early stage of evolution, it will yield new insights for understanding the neurogenesis of vertebrates. First, it will become easy to understand the two different aspects of neural induction, "planer" versus "vertical" inductions (reviewed in Kessler and Melton, 1994). Recent explant experiments using molecular markers clearly indicated that a part of pattern formation of the CNS occurs without vertical signal(s) from mesoderm (Doniach et al., 1992; Ruiz i Altaba, 1992; Blitz and Cho, 1995). This contradicted the traditional view that the latter is essential for induction and pattern formation of anterior CNS. This contradiction can be interpreted as indicating that the fundamental pattern formation of the CNS along the A-P axis may have been established under primitive genetic control without the "vertical" signal(s), and the "vertical" interaction system with underlying mesoderm may have been acquired at a later stage of evolution, making it possible to develop from a primitive brain to the complex brain of the vertebrates.

Second, we would be able to classify various genes involved in pattern formation of the brain by comparing the genetic programs of planarians and other animals, including vertebrates. The most fundamental genes essential for brain development may be understood from the analysis of the developmental program of the planarian brain. We have already shown in the previous paper (Umesono et al., 1997) that the Otx/Otp gene family are involved in pattern formation of the planarian brain. These genes may have been acquired or recruited at an early stage of evolution for brain pattern formation. To understand the evolution of the complex vertebrate brain, it may be important to determine which genes were newly acquired or recruited during brain evolution by comparing genetic programs between planarians and other animals.

\section{ACKNOWLEDGMENTS}

We thank Drs. Tokindo S. Okada, Elizabeth Nakajima, and Ruth Yu for critical reading of the manuscript. We also thank Hidefumi Orii and Takashi Miyata for encouragement. The help of Satoshi Koinuma in the DNA data base search is appreciated. This work was supported by Special Coordination Funds for Promoting Science and Technology to K. A. and Grant-in-Aid for Scientific Research on Priority Areas to K. A. and K. W. 


\section{REFERENCES}

Blitz IL, Cho KWY (1995) Anterior neuroectoderm is progressively induced during gastrulation: the role of the Xenopus homeobox gene orthodenticle. Development 121: 993-1004

Brøndsted HV (1969) Planarian Regeneration. Pergamon Press, Ltd, Oxford, UK

Chitnis A, Henrique D, Lewis J, Ish-Horowicz D, Kintner C (1995) Primary neurogenesis in Xenopus embryos regulated by a homologue of the Drosophila neurogenic gene Delta. Nature 375: 761-766

Doniach T, Phillips CR, Gerhart J (1992) Planer induction of anteroposterior pattern in the developing central nervous system of Xenopus laevis. Science 257: 542-545

Hirth F, Therianos S, Loop T, Gehring WJ, Reichert H, FurukuboTokunaga K (1995) Developmental defects in brain segmentation caused by mutations of the homeobox genes orthodenticles and empty spiracles in Drosophila. Neuron 15: 769-778

Kessler DS, Melton D (1994) Vertebrate embryonic induction: mesodermal and neural patterning. Science 266: 596-604

Li HS, Tierney C, Wen L, Wu JY, Rao Y (1997) A single morphogenic field gives rise to two primodia under the influence of the prechordal plate. Development 124: 603-615

Ouimet T, Mammarbachi A, Cloutier T, Seidah NG, Castelucci VF (1993) cDNA structure and in situ localization of the Aplysia californica pro-hormone convertase PC2. FEBS 330: 343-346

Pigon A (1974) Cephalic mechanisms for the social control of fissioning in planarians. II. Localization and identification of the receptors by electronmicrographic and ablation studies. J Neurobiol 5: $443-462$
Ruiz i Altaba A (1992) Planer and vertical signals in the induction and patterning of the Xenopus nervous system. Development 116: $67-80$

Salivini-Plawen L von (1978) On the origin and evolution of the lower metazoa. Zool Syst Evol 16: 40-88

Sarnat HB, Netsky MG (1985) The brain of the planarian as the ancestor of the human brain. Can J Neurol Sci 12: 296-302

Seidah NG, Fournier H, Boileau G, Benjannet S, Rondeau N, Chretien $M$ (1992) The cDNA structure of the porcine pro-hormone convertase $\mathrm{PC} 2$ and the comparative processing by $\mathrm{PC} 1$ and PC2 of the N-terminal glycopeptide segment of porcine POMC. FEBS 310: 235-239

Simeone A, Acompora D, Gulisano M, Stornaiuolo A, Boncinelli E (1992) Nested expression domains of four homeobox genes in developing rostal brain. Nature 358: 687-690

Thor S (1995) The genetics of brain development: conserved programs in flies and mice. Neuron 15: 975-977

Umesono Y, Watanabe K, Agata K (1997) A planarian orthopedia homolog is specifically expressed in the branch region of both the mature and regenerating brain. Develop Growth Differ 39: 723-727

(Received February 23, 1998 / Accepted March 24, 1998) 